\title{
FORAMINÍFEROS BENTÓNICOS DE SENO GALLEGOS Y BAHÍA BROOKES (54,5 S - 69,5 S), CHILE: PATRONES DE DISTRIBUCIÓN Y DIVERSIDAD
}

\author{
BENTHIC FORAMINÍFERA FROM GALLEGOS SOUND AND BROOKES BAY \\ (54,5 $\left.\mathrm{S}-69,5^{\circ} \mathrm{S}\right)$, CHILE: DISTRIBUTION AND DIVERSITY PATTERNS
}

Tatiana Hromic ${ }^{1} \&$ Américo Montiel ${ }^{2,3}$

\begin{abstract}
This paper describes the distribution pattern and diversity of benthic foraminifera from Gallegos sound and Brookes Bay (XII Region, Chile) and the relationships with the particle size and concentration of the organic matter. Fourteen samples were obtained using a Van Veen grab between 22 and $65 \mathrm{~m}$ water depth in January 2010.

The sediment at all stations was predominantly sand. Thirty-five species of foraminifera were determined from 1601 collected specimens. Mean abundance was 14.8 ind g $^{-1}$ per station. Diversity, measured using Shannon-Wiener index $\left(\mathrm{H}^{\prime}\right)$ varied between 1.7 and 2.8. The highest diversity was recorded in stations at Brookes Bay and the lowest in the stations from Gallegos sound off Ventisquero Garibaldi. Cluster analysis identified two stations groups.

Only sediment texture and particle size were significantly related to mean abundance, diversity and eveness. There was no relationship between depth, concentration of organic matter and the community's parameters. Our results suggest that interstitial space in the sandy habitat play an important role in the establishment of foraminifera communities within Gallegos sound and Brookes Bay.

Key words: benthos, microfauna, meiofauna, fjords, marine periglacial.

1 Laboratorio de Micropaleontología, Instituto de la Patagonia, Universidad de Magallanes, Casilla 113-D, Punta Arenas, Chile. tatiana.hromic@umag.cl

2 Laboratorio de Ecología \& Ciencias Ambientales, Instituto de la Patagonia, Universidad de Magallanes, Casilla 113-D, Punta Arenas, Chile. americo.montiel@umag.cl

3 Fundación Centro de Estudios del Cuaternario Fuego Patagonia y Antártica (CEQUA) Avda. 21 de mayo 1690, Punta Arenas Chile
\end{abstract}




\section{RESUMEN}

Se describe los patrones de distribución y diversidad de los ensambles de foraminíferos bentónicos recolectados en seno Gallegos y bahía Brookes (XII ${ }^{a}$ región, Chile) y su asociación con la granulometría y concentración de materia orgánica del lugar. Las 14 muestras fueron extraídas con una draga Van Veen entre 22 y $65 \mathrm{~m}$ de profundidad durante enero 2010.

El sedimento en todas las estaciones fue dominado por la fracción de arena. En un total de 1.601 ejemplares se identificaron 35 especies de foraminíferos. En promedio se obtuvieron 14,78 ind $\mathrm{g}^{-1}$ por estación. La diversidad, medida a través del índice de Shannon-Wiener, varió entre 1,7 y 2,8. El valor de diversidad más alto se registró en bahía Brookes y el más bajo en seno Gallegos en las estaciones más cercanas al ventisquero Garibaldi. De acuerdo al análisis de conglomerados se clasificaron dos conjuntos de estaciones. La abundancia, diversidad y equidad se relacionaron significativamente con la textura y el tamaño de grano del sedimento. No se determinó ninguna relación entre la profundidad y la concentración de materia orgánica. Esto sugiere que los espacios intersticiales otorgados por el tipo de sedimento juegan un rol clave para las comunidades de foraminíferos del seno Gallegos y bahía Brookes.

Palabras clave: bentos, microfauna, meiofauna, fiordos, periglacial marino.

\section{INTRODUCCIÓN}

Los foraminíferos bentónicos constituyen uno de los componentes relevantes en la base de las cadenas tróficas, por tanto, cualquier alteración en su abundancia, densidad o número de especies afectaría la vida marina (Murray 2006).

El estudio de los foraminíferos en canales y fiordos chilenos es de alta complejidad debido a los numerosos hábitats que ofrece este ecosistema. En consecuencia, es extremadamente difícil establecer patrones generales de distribución espacial. Por tal razón, los estudios de los foraminíferos bentónicos de esta área se han focalizado en determinar el inventario de las especies presentes y algunos datos de abundancia, densidad y número de especies (Zapata \& Alarcón 1988, Zapata \& Moyano 1997, Hromic 1996, 20014, 2002, 2005, 2011a, 2011b, 2011c, 2011d, Marchant 1993). Los valores de abundancia que se han registrado en esta zona han sido muy heterogéneos. Para la zona norte, comprendida entre $42^{\circ}-44^{\circ} \mathrm{S}$, estos oscilaron entre 66 y 431 ind. $\mathrm{g}^{-1}$ y para la zona central, entre $44^{\circ}$ y $47^{\circ} \mathrm{S}$, la abundancia promedio por estación varió entre 10 y 136 ind. $\mathrm{g}^{-1}$. Más al sur, entre $47^{\circ} \mathrm{y}$ $52^{\circ} \mathrm{S}$ la abundancia osciló entre 12 y 72 ind. $\mathrm{g}^{-1} \mathrm{y}$ entre $52-56^{\circ} \mathrm{S}$, la abundancia observada fue de 12

4 Hromic, T. 2001. Foraminíferos bentónicos de canales y fiordos patagónicos chilenos. Expedición Cimar- Fiordo II. Tesis para optar al grado de Magister en Ciencias, Universidad de Concepción. ind. $\mathrm{g}^{-1}$. Finalmente, para el estrecho de Magallanes mismo, se describen entre 3 a 24,9 ind. $\mathrm{g}^{-1}$.

Si bien se han contabilizado 310 especies en el área de canales y fiordos chilenos, más del $80 \%$ de ellas se presentan en unas pocas estaciones y con escasos ejemplares. Entre seno Reloncaví y golfo del Corcovado $\left(42^{\circ}-44^{\circ} \mathrm{S}\right)$ se registraron 74 y 94 especies durante las expediciones CIMAR 10 y CIMAR 11 FIORDOS (ver Informes de crucero, CENDOC: www. cona.cl). Desde boca del Guafo hasta la península de Taitao $\left(44^{\circ}-47^{\circ}\right.$ S) se determinaron 73 y 120 especies (CIMAR 8 y CIMAR 13 FIORDOS) y desde golfo de Penas hasta el estrecho de Magallanes, 88 y 183 especies (CIMAR 2 y CIMAR 14 FIORDOS). Más al sur, desde el Estrecho de Magallanes hasta el cabo de Hornos $\left(52^{\circ}-56^{\circ} \mathrm{S}\right)$ se registraron 170 especies (CIMAR 3 FIORDOS). Otra característica de la foraminiferofauna bentónica de los canales y fiordos chilenos, es el bajo porcentaje de especies que superan el $2 \%$. Por ejemplo, en la zona norte de los fiordos y canales chilenos $\left(42^{\circ}-44^{\circ} \mathrm{S}\right)$ solo llega al 13 y $17 \%$; entre los $44^{\circ}$ y $47^{\circ}$ S, entre el 9 y $15 \%$; entre $47^{\circ}$ y $52^{\circ} \mathrm{S}$, entre 6 y $17 \%$ y finalmente en la zona austral $\left(52^{\circ}-56^{\circ} \mathrm{S}\right)$, sólo el $6,5 \%$ de las especies se encuentran representadas.

Hromic (20035) analizó 14 muestras recolectadas en el estrecho de Magallanes, durante la expedición

5 Hromic, T. 2003.Diversidad y patrones de distribución de foraminíferos bentónicos de canales y fiordos patagónicos al sur del estrecho de Magallanes XXIII ${ }^{\circ}$ Congreso de Ciencias del Mar, Punta Arenas, Chile. 
CIMAR 3 FIORDOS, determinando para el sector de la boca oriental del estrecho un promedio de 15 especies, para la zona de bahía Inútil un promedio de 24 especies y para la zona comprendida entre Cabo Froward y boca occidental un promedio de 31 especies. Por otro lado, en el área marina costera protegida "Francisco Coloane", en la región central del estrecho de Magallanes, Hromic (20086) recolectó 50 muestras, en aguas costaneras someras $k 20$ m) contabilizando un total de 117 especies con un promedio de 22 especies por muestras.

Aún cuando los estudios se han dirigido principalmente a conocer los foraminíferos y sus asociaciones, se han hecho algunos intentos para relacionar este taxón con algunos parámetros ambientales. Ishman \& Martínez (1995) estudiaron los foraminíferos bentónicos de canales y fiordos chilenos, señalando que Cibicides spp. se presentan en ambientes de alta energía; Ehrenbergina pupa y Trifarina angulosa en ambientes con sustratos arenosos y Oridorsalis tener, en aguas con alta salinidad. Violanti et al. (2000) analizaron muestras provenientes del estrecho de Magallanes, documentando que Cibicidoides spp. se relacionan con sedimentos de tipo lodo-arcilloso a profundidades < de $200 \mathrm{~m}$. A pesar de ello, permanece un gran desafío, correlacionar algunos parámetros ambientales, sustrato, disponibilidad de oxígeno, nutrientes, etc. con los agrupamientos o biofacies que se han informado en distintos estudios (Hromic 1999, 2011a, b y c). En este sentido, diversos autores reconocen que los factores que más influyen en la distribución de los foraminíferos bentónicos son: tipo de sustrato y profundidad (Hayward et al. 2002), disponibilidad de oxígeno y nutrientes (Boltovskoy et al. 1980) y en menor grado la temperatura, salinidad y profundidad.

De especial interés para los investigadores es el conocimiento del tipo y comportamiento de la fauna al interior de los fiordos patagónicos, cuyo ambiente tiene gran influencia de los Campos de Hielo aledaños. En muchos de ellos, se observa la presencia de glaciares, con sedimentos dominados por sustratos muy blandos (Silva et al. 2001; Silva \& Ortiz 2002), con alta tasa de sedimentación (Hoskin

6 Hromic,T. 2008. Foraminíferos bentónicos como indicadores biológicos de ambientes costeros, del Área Marina Costera Protegida Francisco Coloane, Magallanes, Chile XXVIII ${ }^{\circ}$ Congreso de Ciencias del Mar, Viña del Mar, Chile
\& Barrel 1972), y con condiciones estuarinas (Siever \& Silva 2008), causadas por efecto del deshielo (Vásquez 20117).

El presente trabajo tiene como objetivo determinar el patrón de distribución espacial de los foraminíferos y su relación con las características del sedimento al interior del seno Gallegos y Bahía Brookes (seno Almirantazgo, XII ${ }^{\underline{a}}$ Región, Chile), cercanos al Campo de Hielo de la cordillera Darwin.

\section{MATERIALES Y MÉTODOS}

Área de estudio

Seno Gallegos (54 28' 58's; 69 50' $\left.51,88^{\prime \prime} \mathrm{W}\right)$ se ubica al interior de bahía Brookes (54²3'; 37,04'S; 69 50'; 48,92'"W). En el extremo suroeste de dicho seno se ubica el Glaciar Garibaldi, el cual se desprende del Campo de Hielo de la Cordillera Darwin (Fig. 1). Oceanográficamente, Vásquez $\left(2011^{7}\right)$ señala la presencia un cuerpo de agua con dos capas de agua, uno superficial (hasta $25 \mathrm{~m}$ de profundidad) caracterizado por temperaturas entre $3,7^{\circ} \mathrm{C}$ y $9{ }^{\circ} \mathrm{C}$, salinidad con rango entre 23,56 y 30,06 PSU, y concentraciones de clorofila a que oscilan entre $<1$ y $15,41 \mathrm{mg} \mathrm{m}^{-3}$,y otro profundo (bajo los $25 \mathrm{~m}$ de profundidad), que presenta un rango de 6,06 - 7,19 ${ }^{\circ} \mathrm{C}$ para temperatura, 29,45 - 30,13 PSU de salinidad, $y<1 \mathrm{mg} \mathrm{m}^{-3}$ para la concentración de clorofila $a$.

\section{Trabajo de terreno}

Las muestras de sedimento fueron obtenidas tanto en seno Gallegos como en Bahía Brookes, entre el 5 y 10 de enero del 2010 (verano austral) a bordo del L/M Cabo Tamar. Estas muestras fueron recolectadas mediante una draga Van Veen $\left(0,1 \mathrm{~m}^{2}\right)$. En total se obtuvo material en siete estaciones de muestreo en cada una de las cuales se recolectó 2 muestras para fauna foraminiferológica $(n=14)$ y una muestra para análisis granulométrico del sedimento. Las estaciones GAL 1, 2 y 3 se localizaron en las zonas más cercanas al Ventisquero Garibaldi mientras que las estaciones GAL 3, 4 y 5, se ubicaron en la

7 Vásquez, O. 2011. Evaluación espacial y temporal de la influencia de un glaciar sobre características oceanográficas en seno Gallegos (seno Almirantazgo, Chile). Tesis de grado en Biología Marina. Universidad de Magallanes. 


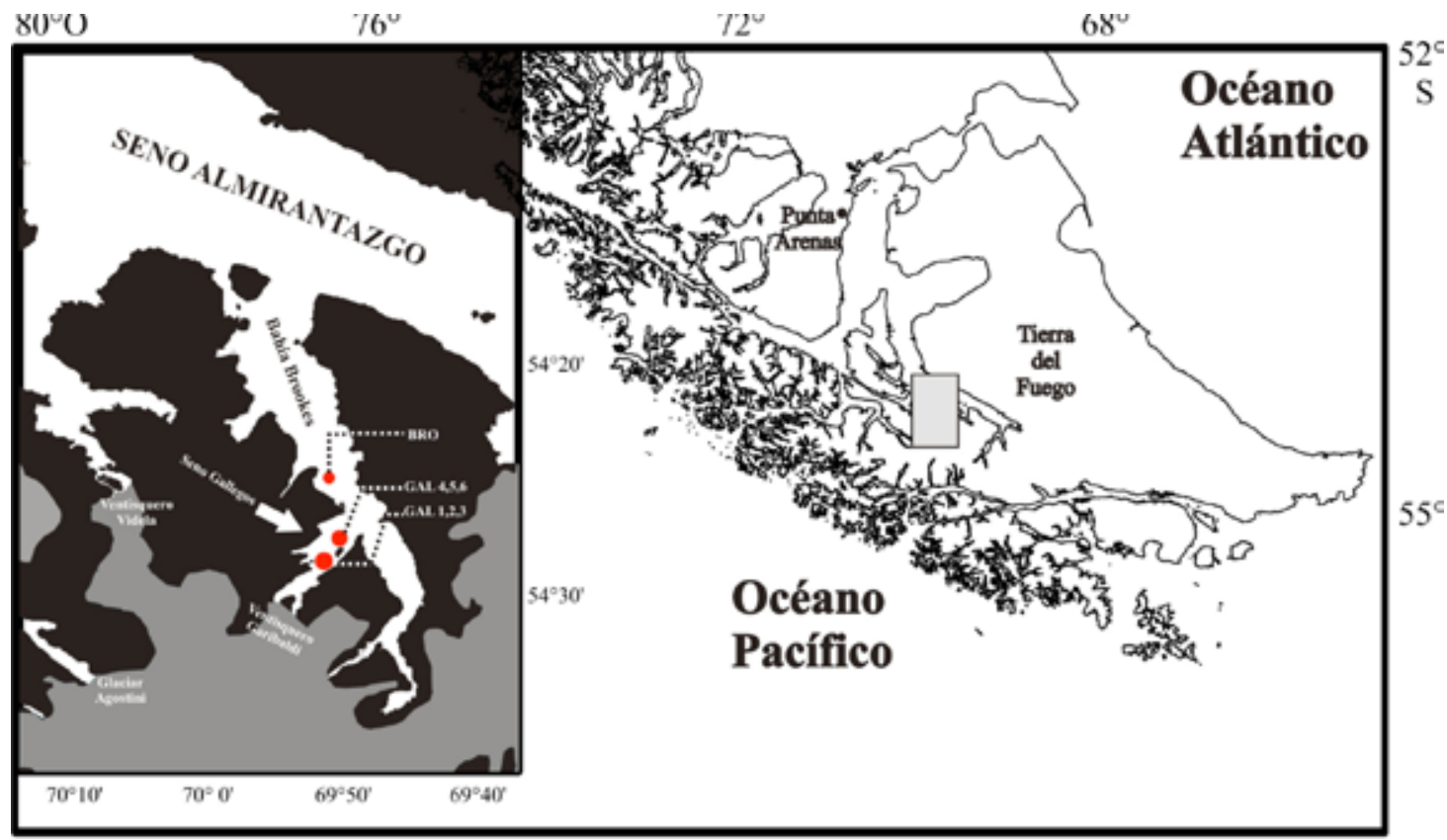

Fig. 1. Mapa del área de estudio, señalando los lugares desde los cuales se extrajeron las muestras.

boca del seno Gallegos. La estación BRO se situó en las inmediaciones de bahía Brookes, fuera del seno Gallegos (Fig. 1). Debido a las características sedimentológicos del área de estudio, las muestras fueron obtenidas a distintas profundidades. Para la obtención de los foraminíferos se apartaron cuidadosamente los primeros 5 centímetros de cada muestra de sedimento. Seguidamente, las muestras para análisis faunístico y granulométrico fueron depositadas en bolsas de polietileno y conservadas a temperatura ambiente.

Análisis de laboratorio

Para la caracterización granulométrica de cada estación de muestreo, el sedimento se secó durante 24 hrs. El material seco se tamizó en un cribador vibratorio eléctrico, utilizando una batería de 6 tamices (3,35 mm, $1 \mathrm{~mm}, 500 \mathrm{~mm}, 250 \mathrm{~mm}$, $125 \mathrm{~mm}, 63 \mathrm{~mm}$ y una bandeja para los sedimentos finos). Siguiendo el método propuesto por Folk (1974) se determinaron los parámetros estadísticos $\left(\operatorname{Phi}(\Phi)=-\log _{2}\right.$ de diámetro de partícula $(\mathrm{mm})$ ). Las muestras se clasificaron en clases texturales utilizando el diagrama de Shepard y el programa Triplot 1.4 (Graham \& Midgley 2000). Se determinó el contenido de materia orgánica (MOT) mediante la técnica de pérdida por ignición $450^{\circ}$ por 4 hrs, siguiendo el método descrito por Dean (1974).

Para la separación de los caparazones de foraminíferos, las fracciones superficiales de los sedimentos fueron reducidas lavando los sedimentos a través de un tamiz de $63 \mu$. El sedimento retenido en el tamiz fue secado y pesado. Seguidamente, de cada muestra se extrajo, utilizando un estéreomicroscopio (Wild Heerbrugo $®)$, uno a uno todos los foraminíferos totales. A continuación los ejemplares de foraminíferos fueron depositados en reglillas, determinados a nivel de especie y contados (Boltovskoy 1965).

La identificación hasta nivel genérico se basó en los trabajos de Loeblich \& Tappan (1988) y Decrouez (1989). La determinación específica se efectuó de acuerdo al catálogo de Ellis \& Messina (1944 et seq.) y a la literatura del área, principalmente los trabajos de Brady (1884), Baker (1960), Zapata \& Moyano (1997), Boltovskoy et al. (1980) y Hromic $\left(2001^{4}\right)$. Conjuntamente, los foraminíferos se contabilizaron de acuerdo al tipo de caparazón: arenáceo o calcáreo.

Análisis de los datos

A partir de valores de abundancia absoluta (ejemplares/peso de la muestra), se calculó densi- 


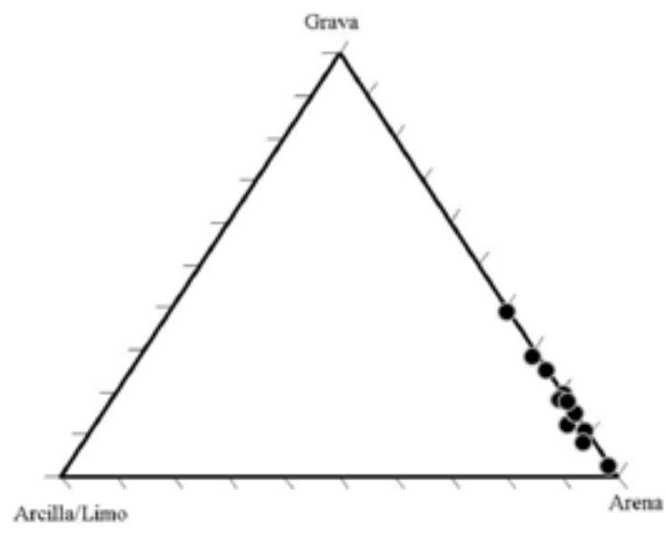

Fig. 2. Posición de las muestras en diagrama de clasificación textural de sedimentos de Shepard. Modificado de Folk (1980).

dad de foraminíferos estandarizando el número de ejemplares por gramo de muestra $\left(e \mathrm{j} . \mathrm{g}^{-1}\right)$. Además, se contabilizó el número de especies (S) y se calculó los índices diversidad de Shannon-Wiener $\left(\mathrm{H}^{\prime}\right)$ y de equidad $\left(J^{\prime}\right)$ para cada estación de muestreo.

Para determinar el posible patrón de distribución espacial en el área de estudio, se aplicó la técnica multivariada de agrupamiento (cluster) basado en los valores de densidad $\left(e \mathrm{j} . \mathrm{g}^{-1}\right)$. Estos valores fueron estandarizados, previa aplicación del índice de similitud de Jaccard. La significancia estadística de las agrupaciones obtenidas con el análisis de agrupamiento se evaluó con la rutina SIMPROF (similarity profile; Clarke \& Warwick 2001) al 95\% de confianza, bajo la hipótesis nula de que no hay diferencias en la composición de foraminíferos entre las estaciones de muestreo. La contribución de las especies/taxa a la diferenciación o similitud entre grupos se analizó con la rutina SIMPER (similarity percentages; Clarke 1993).
Tanto los análisis multivariados como el cálculo de índices se realizaron con el programa PRIMER 6.0 (Clarke \& Gorley 2006).

Para determinar la relación entre la fauna foraminiferológica y las variables abióticas se aplicó un análisis de regresión lineal $(\alpha=0,05)$. Previo a la aplicación de este análisis, los valores porcentuales de grava, arena, limo - arcilla y MOT fueron transformados usando el arcoseno (x) (Zar, 1999). Esta prueba estadística se calculó utilizando el programa StatView 5.0.

\section{RESULTADOS}

\section{Características sedimentológicas}

A partir del análisis granulométrico se determinó que los porcentajes de arena-guijarrosa variaron entre 71,4 y 93 \%. En general, el sedimento fue pobremente seleccionado y dominado por la fracción de arena (Fig. 2). En la estación GAL 1 la fracción dominante fue la arena media $(\Phi=1$ - 2) mientras que en las estaciones GAL 2, 3 y 5, los sedimentos se caracterizaron por presentar una alta dominancia de arena gruesa $(\Phi=0$ - 1) y la estación ubicada en el bahía Brookes, el sedimento dominante fue la arena fina $(\Phi=1-2)$ (Tabla 1$)$.

El contenido de materia orgánica en los sedimentos fue bajo y varió entre 2,83\% (GAL3) y $0,79 \%$ (GAL6). Las mayores concentraciones se obtuvieron en las estaciones más cercanas al ventisquero Garibaldi en el seno Gallegos, mientras las menores concentraciones fueron obtenidas en la desembocadura del seno Gallegos hacia bahía Brookes (Tabla 1).

Tabla 1. Lista de estaciones y sus respectivos parámetros comunitarios ( $\mathrm{D}=$ Densidad, S = número de especie; H'= Índice Shannon - Wiener; J'= Índice de equidad Pielou) y de los sedimentos (ni = sin información)

\begin{tabular}{|c|c|c|c|c|c|c|c|c|c|c|c|c|c|c|}
\hline Estación & $\begin{array}{c}\text { Fecha } \\
2010\end{array}$ & Localidad & $\begin{array}{l}\text { Lat S } \\
\text { o }, "\end{array}$ & 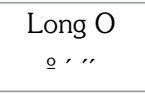 & $\begin{array}{c}\text { D } \\
\text { Ind/g. }\end{array}$ & S & J' & $\begin{array}{c}\mathrm{H}^{\prime} \\
\left(\log _{e}\right)\end{array}$ & $\begin{array}{c}\text { Prof. } \\
\text { m. }\end{array}$ & $\begin{array}{c}\text { MOT } \\
(\%)\end{array}$ & $\begin{array}{c}\text { Grava } \\
(\%)\end{array}$ & $\begin{array}{c}\text { Arena } \\
(\%)\end{array}$ & $\begin{array}{c}\mathrm{L}+\mathrm{A} \\
(\%)\end{array}$ & $\begin{array}{l}\text { Phi } \\
(\Phi)\end{array}$ \\
\hline GAL1 & 09.01 & Seno Gallegos & 542906.7 & 695118.9 & 10,38 & 23 & 0,8 & 2,6 & 22 & 2,41 & 6,5 & 93,1 & 0,4 & $1,46 \pm 1,28$ \\
\hline GAL2 & 09.01 & Seno Gallegos & 542907.8 & 695103.3 & 24,67 & 21 & 0,6 & 1,9 & 37 & 2,33 & 21,5 & 76,9 & 1,6 & $0,69 \pm 1,64$ \\
\hline GAL3 & 09.01 & Seno Gallegos & 542913.8 & 695054.7 & 14,26 & 14 & 0,8 & 2,0 & 60 & 2,83 & 14,1 & 85,6 & 0,3 & $0,66 \pm 1,53$ \\
\hline $\mathrm{BRO}$ & 10.01 & Bahía Brookes & 542559.6 & 695103.5 & 6,58 & 29 & 0,8 & 2,8 & 56 & 1,07 & 0 & 90,2 & 9,8 & $2,30 \pm 1,68$ \\
\hline GAL4 & 11.01. & Seno Gallegos & 542830.2 & 694932.2 & 8,91 & 16 & 0,9 & 2,4 & 20 & 0,87 & ni & $\mathrm{ni}$ & ni & \\
\hline GAL5 & 11.01. & Seno Gallegos & 542839.8 & 695017.8 & 34,55 & 15 & 0,6 & 1,7 & 65 & 2,31 & 27,8 & 71,4 & 0,8 & $0,27 \pm 1,37$ \\
\hline GAL6 & 11.01. & Seno Gallegos & 542842.3 & 695014.2 & 4,13 & 18 & 0,8 & 2,3 & 43 & 0,79 & ni & ni & ni & \\
\hline
\end{tabular}




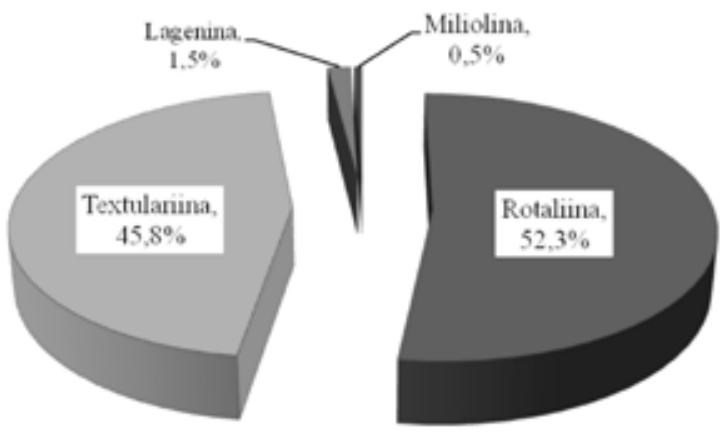

Fig. 3. Abundancia relativa de los subórdenes de foraminíferos bentónicos presentes en seno Gallegos/ bahía Brookes.

Composición taxonómica por suborden

En total se determinaron 35 especies de foraminíferos, pertenecientes a 4 subórdenes: Textulariina, Rotaliina, Lagenina y Miliolina (Anexo 1). En términos de abundancia, el suborden con mayor representación fue Rotaliina con 9 géneros, 10 especies y con el 52,3 \% de los ejemplares. Le siguió Textulariina con 8 géneros, 18 especies y con el 45,8 \% de los ejemplares, Lagenina con 3 géneros y 6 especies y Miliolina, con 1 género y una especie, los que contribuyeron con escaso porcentaje de ejemplares, 1,5 y $0,5 \%$, respectivamente (Fig. 3). En las estaciones GAL 5 y 6 , el suborden Textulariina presentó porcentajes sobre el 50\%. Mientras que en las demás estaciones, el suborden Rotaliina fue dominante (> 50\%), especialmente en la estación GAL 2 en donde este suborden alcanzó el 73,4\% (Fig. 4).

Características ecológicas de los foraminíferos

El número total de especímenes recolectados fue de 1.601 foraminíferos bentónicos equivalente a 239,5 ej. $\mathrm{g}^{-1}$.

Sólo un tercio (34,3\%) de las especies identificadas superó el $2 \%$ de abundancia. Estas 12 especies conformaron el 90,7\% del total de ejemplares. Las especies dominantes fueron Nonionella auris $(18,8$ $\%)$, Globobulimina notovata (16,8 \%) y Labrospira jeffreysii (12,6\%) (Tabla 2). Respecto de la frecuencia, el 64,7\% de las especies presentan una distribución más bien restringida, encontrándose en 1 (a 4) estaciones, (71,4\%). Tal es el caso de Ammodiscus flavidus, Reophax pilulifer y Glandulina communis. El 14,7\% de las especies se encontraron en 5 (a 6) estaciones (85\%) e.g. Labrospira kosterensis y sólo el $21 \%$ del total de especies, se encontró en todas las estaciones (Fig. 5). Las especies que se distribuyeron ampliamente en el área de estudio fueron Ammobaculites americanus, Labrospira jeffreysii, Rhabdammina abyssorum, Globobulimina notovata, Nonion scaphum, Nonionella auris y Uvigerina bifurcata.

En relación a la densidad, destaca el hecho de que 6 especies conformaron el $73 \%$ de la microfauna. Los valores de densidad variaron entre 34,6 ej. $\mathrm{g}^{-1}$ y 4,1 ej. $\mathrm{g}^{-1}$, registrados en las estaciones GAL

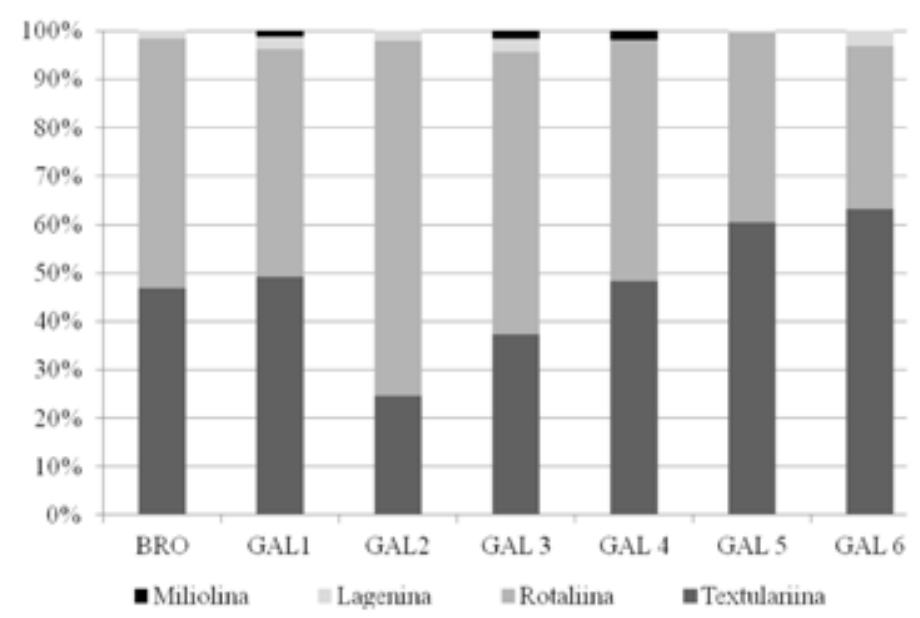

Fig. 4. Contribución porcentual de cada suborden de foraminíferos bentónicos por estación de muestreo, seno Gallegos/ bahía Brookes. 


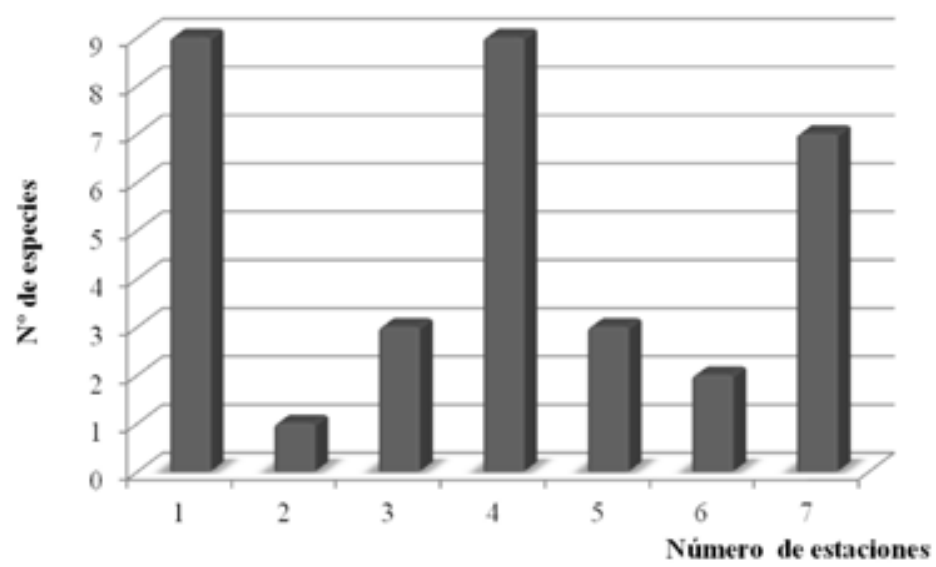

Fig. 5. Distribución geográfica: Número de especies en relación al número de estaciones en que se encuentran.

5 (65 m) y GAL $6(43 \mathrm{~m})$ respectivamente, situadas ambas en la boca del seno Gallegos (Tabla 1, Fig. 6).

Del total de especies de foraminíferos, el 51,4 \% (18 especies), presentaron caparazón arenáceo, mientras que las restantes especies presentaron caparazón calcáreo. En la localidad GAL 2 (37 m), más cercana al glaciar del seno Gallegos, el total de organismos con caparazón arenáceo alcanzó el $24,7 \%$ de los ejemplares, llegando a un máximo del 63,2\% en la localidad GAL 6 (43 m), en la boca del seno Gallegos. En cambio, los foraminíferos con caparazón calcáreo, se encuentran representados en porcentajes superiores al 36,8 \%, dominando claramente solo en la localidad GAL 2 (37 m) $(75,4$ \%) (Tabla 3).
Tabla 2: Representación porcentual de las especies más abundantes (> 2\%) en el área de muestreo.

\begin{tabular}{lc}
\hline Especies & $\%$ \\
\hline Nonionella auris & 18,8 \\
Globobulimina notovata & 16,8 \\
Labrospira jeffreysii & 12,6 \\
Ammobaculites americanus & 10,5 \\
Nonion scaphum & 8,6 \\
Rhabdammina abyssorum & 5 \\
Labrospirasp. 1 & 4,3 \\
Morulaeplecta bulbosa & 3,9 \\
Uvigerina bifurcata & 3,5 \\
Buccella peruviana s.l. & 2,8
\end{tabular}

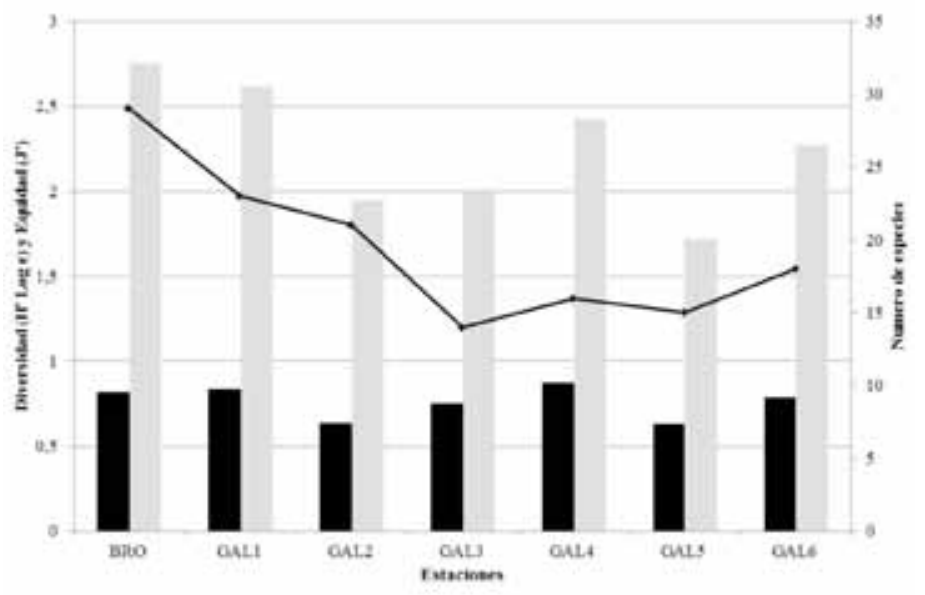

Fig. 6. Número de especies (línea negra), diversidad (índice de Shannon-Wiener, barra gris) y equidad (Índice de Pielou, barra negra) por estación de muestreo en seno Gallegos/ bahía Brookes. 
Tabla 3: Relación porcentual entre foraminíferos arenáceos y calcáreos por estación (en negrita se destacan las localidades con mayor porcentaje de foraminíferos arenáceos y calcáreos) .

\begin{tabular}{lccccccc}
\hline & BRO & GAL2 & GAL 1 & GAL 6 & GAL 3 & GAL 4 & GAL 5 \\
\hline \% arenáceos & 46,9 & 24,6 & 49,2 & $\mathbf{6 3 , 2}$ & 37,2 & 48,4 & 60,4 \\
\% calcáreos & 53,1 & $\mathbf{7 5 , 4}$ & 50,8 & 36,8 & 62,8 & 51,6 & 39,6 \\
\hline
\end{tabular}

Biodiversidad de foraminíferos

El valor más alto de especies (29 especies) se registró en bahía Brookes (56 m) y el más bajo (14 especies) fue encontrado en la estación GAL 3 en seno Gallegos (60 m) (Tabla 1). El índice de diversidad Shannon-Wiener varió entre 1,7 y 2,8 . El valor más alto se registró en la bahía Brookes (BRO) y el más bajo en la estación GAL 5, seno Gallegos frente al glaciar (65 $\mathrm{m}$ profundidad). En promedio el valor de equidad $\left(\mathrm{J}^{\prime}\right)$ fue de 0,75 . La estación GAL $4(20 \mathrm{~m})$ presentó el mayor valor de $\mathrm{J}^{\prime}(0,9)$ y las estaciones GAL 2 y 5 fueron las que presentaron los menores valores $(0,6$; Tabla 1$)$.

Patrón de distribución

De acuerdo a los análisis de similitud y SIMPROF se separaron significativamente dos conglomerado: el conglomerado A que incluyó las estaciones GAL 3 (frente al glaciar, seno Gallegos) y GAL 4 (boca del seno Gallegos) y el conglomerado B que incluyó las estaciones GAL 1, GAL 2 (ambas frente al glaciar, al interior del seno Gallegos), GAL 6 (boca del seno Gallegos) y BRO (bahía Brookes). En ambos grupos se mezclaron muestras obtenidas desde diferentes profundidades (20, 40 y $60 \mathrm{~m})$. La estación GAL 5 (boca del seno Gallegos) segregó de los dos conglomerados (Fig.7).

A partir del análisis SIMPER se determinó que el promedio de dismilitud entre los ensambles fue de un $52,4 \%$. Además, las especies que contribuyeron a las diferencias entre los ensambles A: Brookes (BRO) y seno Gallegos (GAL 1, GAL 2 y GAL 6) y B: (GAL 3 y GAL 4) fueron Nonionella auris $(15,7 \%)$, Labrospira jeffreysii (11,4\%), Buccella peruviana s.l. (8\%), Nonion scaphum (7\%), Trochammina discorbis $(5,4 \%)$ y Rhabdammina abyssorum (5,2\%) (Tabla 4).

Relación entre características comunitarias de foraminíferos y variables abióticas

Se determinó una relación significativa entre la densidad (ind. $\mathrm{g}^{-1}$ ) y la fracción de grava y arena $\left(r^{2}=0,8-0,9 ; p<0,05\right)$. El número de especies, en cambio, sólo mostró una relación significativa con el

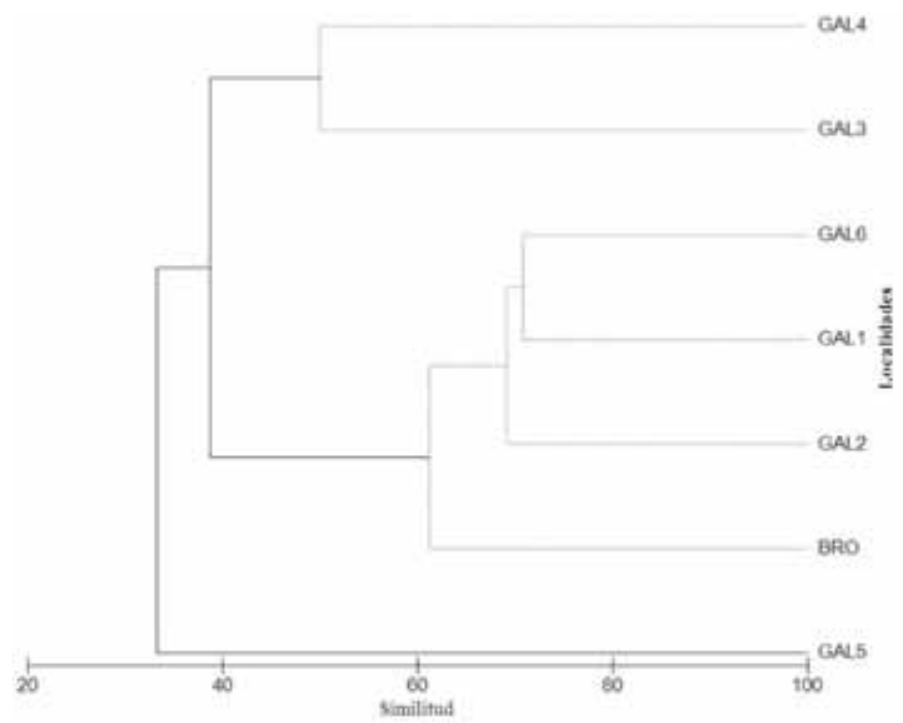

Fig. 7. Dendrograma realizado a partir de la matriz de similitud de Jaccard para los foraminíferos bentónicos. Las diferencias significativas $(p<0,05)$ entre conglomerados obtenidas a partir del análisis SIMPROF se presentan con líneas negras en el dendrograma. 
Tabla 4. Representación porcentual de las especies que contribuyen a la segregación de los dos conglomerados.

\begin{tabular}{lcc}
\hline $\begin{array}{l}\text { Promedio similitud } \\
\text { entre A \& B= 52,4\% }\end{array}$ & $\begin{array}{c}\text { Contribución } \\
\%\end{array}$ & $\begin{array}{c}\text { Acumulativo } \\
\%\end{array}$ \\
Especies & 15,7 & 15,7 \\
\hline Nonionella auris & 11,4 & 27,1 \\
Labrospira jeffreysii & 8,0 & 35,1 \\
Buccella peruviana s.l. & 6,9 & 42,1 \\
Nonion scaphum & 5,4 & 47,5 \\
Trochammina discorbis & 5,2 & 52,7 \\
Rhabdammina abyssorum & 4,6 & 57,3 \\
Morulaeoplecta bulbosa & 4,4 & 61,6 \\
Ammobaculites americanus & 4,1 & 65,7 \\
Labrospira sp. 1 & 3,4 & 69,7 \\
Globobulimina notovata & 3,6 & 73,2 \\
Remaneica helgolandica & 3,4 & 76,7 \\
Reophax pilulifer & 3,00 & 79,7 \\
Angulogerina angulosa & 2,7 & 82,4 \\
Uvigerina bifurcata & 2,6 & 85,0 \\
Ammodiscus flavidus & 2,5 & 87,5 \\
Reophax scorpiurus & 2,1 & 89,7 \\
Labrospira kosterensis & 1,4 & 91,00 \\
Quinqueloculina seminula & &
\end{tabular}

diámetro de grano $\left(\mathrm{r}^{2}=0,8 ; \mathrm{p}<0,05\right)$. La diversidad mostró una relación significativa con la grava, arena y diámetro de grano $\left(\mathrm{r}^{2}=0,8-0,8-0,9\right.$ respectivamente; $p<0,05)$. Finalmente el índice de equidad Pielou sólo se relacionó significativamente con la fracción de arena $\left(\mathrm{r}^{2}=0,9 ; \mathrm{p}<0,05\right)$. En contraste, no se encontró ninguna relación entre los parámetros comunitarios con la profundidad y la concentración de materia orgánica (Tabla 5).

\section{DISCUSIÓN}

A pesar de que en las últimas décadas se han realizado numerosos estudios sobre el inventario de los foraminíferos de la región de canales y fiordos chilenos (Marchant 1993, Hromic 1998, 1999, 2001, 2001, 2002, 2005, 2007, 2009a y 2009b, 2011a, 2001b, 2011c, 2011d, Hromic \& Zúñiga 2003, 2005, Hromic et al. 2006, Zapata \& Alarcón 1988, Zapata \& Moyano 1997, Zapata et al. 1995), en lo que respecta a la diversidad y su relación con las variables abióticas las investigaciones aún son escasas. Por otro lado, en la región de Magallanes existen grandes desigualdades en términos del esfuerzo de muestreo, generando áreas bien estudiadas y áreas sin ningún tipo de antecedente. En este contexto, los resultados que se han descrito en este trabajo representan la primera información sobre los foraminíferos que habitan en un área periglaciar marina, como lo es el seno Gallegos y la bahía Brookes.

A nivel de número de subórdenes, la foraminíferofauna del seno Gallegos y bahía Brookes es menos diversa que en el resto de los canales y fiordos patagónicos, para los cuales se citan 6 subórdenes (Hromic 20014). Es notable la ausencia de robertínidos, espirillínidos y el escaso número de especies de miliólidos, que en el resto de los canales alcanzan al 2,7 \%, 0,5\% y $13 \%$, respectivamente (Hromic op. cit.), lo cual indicaría que estos taxa presentan dificultades para penetrar al interior de estas áreas periglaciares. En las estaciones donde dominan los rotálidos, estos en general sobrepasan levemente el $50 \%$ de representación. El porcentaje del suborden Textulariina, que en este estudio oscila entre 24,6 y $63,2 \%$, es bastante más alto a lo observado en la zona de canales y fiordos, en donde se encuentra en abundancias inferiores al 20\% (Zapata \& Moyano 1997, Violanti et al. 2000, Hromic et al. 2006, Hromic 2009a, 2009b, 2011a, b, c y d (en prensa). Específicamente en la zona sud-austral chilena $\left(52^{\circ}-56^{\circ}\right.$ S) llega al 16,5\% (Hromic 2011b, en este volumen), mientras que en el área norte de los canales (Golfo Ancud, Corcovado y Reloncaví) llega al 29\% (Hromic 2011d, en prensa). Las especies pertenecientes a este suborden, poseen caparazón arenáceo y son propias de ambientes estuarinos

Tabla 5. Coeficiente de correlación $\left(\mathrm{r}^{2}\right)$ obtenido mediante el análisis de regresión lineal entre las características del sedimento (L+A = limo y arcilla) y los parámetros comunitarios $(\mathrm{D}=$ Densidad, $\mathrm{S}=$ número de especie; H'= Índice Shannon-Wiener; J' Índice de equidad Pielou). En negritas valores significativos $(\leq 0,05)$.

\begin{tabular}{|c|c|c|c|c|c|c|}
\hline & $\begin{array}{c}\text { Prof. } \\
\text { m. }\end{array}$ & Grava & Arena & $\mathrm{L}+\mathrm{A}$ & MOT & $\begin{array}{l}\text { Phi } \\
(\Phi)\end{array}$ \\
\hline $\mathrm{D}\left(\right.$ Ind $\left.\mathrm{g}^{-1}\right)$ & 0,2 & 0,8 & 0,9 & 0,2 & 0,4 & 0,7 \\
\hline S & 0,02 & 0,7 & 0,4 & 0,6 & 0,05 & 0,8 \\
\hline $\mathrm{H}^{\prime}\left(\log _{e}\right)$ & 0,2 & 0,8 & 0,8 & 0,3 & 0,3 & 0,9 \\
\hline J' & 0,3 & 0,7 & 0,9 & 0,08 & 0,3 & 0,6 \\
\hline
\end{tabular}


(Murray 1991), generado por la confluencia de aguas marinas con aguas procedentes del glaciar (Vásquez 20117). El aumento de formas arenáceas en zona próximas a glaciares fue descrita también en fiordos del hemisferio norte por Korsum \& Hald (2000), quienes lo atribuyeron a la mezcla estacional de aguas frías o a la alta tasa de sedimentación.

En general, la composición taxonómica de los foraminíferos identificados en este estudio es común en el área de canales sud-austral chilena (Marchant 1993, Hromic 1999, 20014, 2002, 2005, 2007, 2009a y 2009b, 2011a, 2001b, 2011 c, 2001 d (en prensa) Hromic \& Zúñiga 2003, 2005, Hromic et al. 2006, Zapata \& Alarcón 1988, Zapata \& Moyano 1997, Zapata et al. 1995). No obstante, la ausencia de especies pertenecientes a los géneros Cibicidoides, Globocassidulina y Trifarina, dominantes en las asociaciones del estrecho de Magallanes (Violanti et al., 2000) permite inferir que el ambiente de seno Gallegos y bahía Brookes es de menor energía, en relación al Estrecho, lo cual podría asociarse a una circulación con menores velocidades de corrientes. Las especies de género Cibicidoides, se adhieren al sustrato y son frecuentes en ambientes turbulentos (Ishman \& Martínez 1995). Otra especie ausente es Ammonia beccarii, caracterizada por ser euritolerante y ser común en aguas hiposalinas, esta especie se presenta en forma abundante en aguas someras del canal Beagle (Lena 1966). Su ausencia sugiere que en el area de estudio el ambiente presenta características más marinas que en otras áreas de los canales.

En términos de abundancia, los valores calculados para el seno Gallegos y bahía Brookes son bajos en comparación con los registrados para el área sud-austral chilena, los que llegan a un promedio de 265,4 ej. $\mathrm{g}^{-1}$ (Hromic, $2011 \mathrm{~b}$ en este volumen) y que han llegado más al norte a los 986 ej. $\mathrm{g}^{-1}$ (Hromic $2011 d$ en prensa). Sin embargo, los valores obtenidos en este estudio son más altos que los reportados para la sección del estrecho de Magallanes, en donde se cita un promedio de 3 ej. $\mathrm{g}^{-1}$ para el área de la boca oriental, 4,9 ej. $\mathrm{g}^{-1}$ para el sector de bahía Inútil y de 24,9 ej. $\mathrm{g}^{-1}$ para la zona entre Cabo Froward y boca occidental del Estrecho (Hromic 20035). La zona oriental y bahía Inútil del estrecho de Magallanes tendrían una menor cantidad de foraminíferos en relación con Gallegos y Brookes, mientras que la zona occidental se acercaría más a estos valores, lo que sugiere en forma preliminar que en Gallegos y Brookes hay un menor estrés ambiental que en la zona oriental de estrecho de Magallanes.

Respecto de la diversidad, el número de especies registradas en el estrecho de Magallanes va aumentando desde la boca oriental a la occidental $(15,24$ y 31 especies promedio para las áreas antes mencionadas) mientras que en Gallegos se contabilizaron 19 especies promedio para la zona frente al glaciar, 16,3 para la zona cercana a la boca y para Brookes 29 especies, siendo similar a la zona occidental del Estrecho. Estos resultados sugieren que dentro del seno Gallegos y bahía Brookes se genera un ambiente con mayor influencia de aguas oceánicas, que probablemente se relacionen más con los aportes del lado occidental del Estrecho (aguas del Pacífico y antárticas) que con las provenientes del Atlántico. Violanti et al. (2000) mapearon las asociaciones faunísticas, indicando la abundante presencia del género Cibicides en el área oriental del Estrecho. La ausencia de este género en Gallegos y Brookes avalaría la idea de un mayor aporte de aguas desde el Pacífico y además aguas menos energéticas, puesto que éstas son especies que viven adheridas al sustrato y que tipifican ambientes turbulentos (Ishman \& Martínez 1995). La presencia de Nonionella auris y otras formas infaunales como G. notovata y $U$. bifurcata sugiere además la presencia de ambientes más bien pobres en oxígeno o micróxicos (Sen Gupta \& Machain Castillo 1993, Páez \& Zúñiga 2001) y probablemente con menor disponibilidad de nutrientes lo que sin duda también incide en la abundancia y densidad. De modo general, Gallegos y Brookes tendrían un mayor recambio de aguas que la observada en otros fiordos patagónicos, en donde claramente la abundancia (densidad) y la diversidad es menor (Hromic 20014, Hromic et al. 2006) pero no tan extenso como sucede en el propio estrecho. Ahora bien es probable que el flujo tenga la suficiente fuerza para impedir el depósito de sedimentos muy finos, predominando arenas y grava, lo que obligaría a especies como $N$. auris, típica de ambientes fangosos y de baja energía (Murray 1991) a adaptarse a sustratos más gruesos.

En relación a los resultados obtenidos en el análisis de conglomerados, exceptuando la fauna de la estación GAL 5, todo el resto de las estaciones se encuentra por sobre el $50 \%$ de similitud, lo que indica la presencia de un ensamble común para el área, que se subdivide en dos grupos. Esta separación se 
puede atribuir a diferencias faunísticas producida por heterogeneidad de microhábitats, que son comunes de observar en canales y fiordos (Hromic, 20014). La similitud que se observa entre las estaciones de Brookes y las más cercanas a la cabeza del seno Gallegos se podría explicar por el transporte de las especies a causa de la circulación estuarina que es característica de los fiordos australes (Silva, 2008).

Respecto de los factores que intervienen en la distribución, el tipo de sustrato es más importante que el MOT para regular la diversidad al interior de Gallegos y bahía Brookes. Específicamente la presencia de partículas de mayor tamaño como gravas y arenas mostró una fuerte correlación con este parámetro ( 0,8 y 0,9 respectivamente), mientras que el número de especies se correlacionó con Phi y no se estableció (o muy baja) ninguna correlación con MOT. En este sentido, algunos autores (Mackensen et al. 1995, Schmiedl et al. 1997, fide Haslett 2002), sugieren que la distribución a escala global de los foraminíferos depende sustancialmente de la ventilación, que afectaría a la disponibilidad de oxígeno y temperatura, de la productividad, de los aportes de materia orgánica y del $\mathrm{pH}$, otros autores sugieren, que a escala regional es una combinación de factores, entre los cuales no es menor la importancia de las características sedimentológicas, específicamente tipo de sedimento y tamaño de partículas (Smart 2002).

En este estudio se observa como la fracción gruesa de partículas tiene mayor relevancia que la materia orgánica total MOT, las partículas pequeñas y la profundidad, factores con los cual se estableció una correlación muy baja, no significativa. Los foraminíferos utilizarían los espacios intersticiales de las partículas más grandes, para establecerse. El índice de Pielou también se correlacionó significativamente con el sustrato arenoso, lo que respalda la importancia del tamaño de partícula como factor limitante. Los taxones más abundantes identificados en esta oportunidad, como Ammobaculites, Nonionella, Nonion, Globobulimina, Buccella, Reophax, etc. son todos de hábitos infaunales (Murray 1991), es decir, aprovechan estos intersticios, lo que concuerda con los resultados obtenidos.

\section{AGRADECIMIENTO}

Se agradece al capitán Alejandro González y a la tripulación del L/M Cabo Tamar por el apoyo en el desarrollo del trabajo de terreno. Al Biólogo
Marino Osvaldo Vásquez por el trabajo laboratorio en granulometría. A la Srta. Lyta Quezada, por su aporte en la extracción de los foraminíferos. Este artículo fue financiado por el proyecto FONDECYT iniciación $\mathrm{N}^{\circ} 11090208$ (A. Montiel). Los autores desean agradecer también a los árbitros anónimos por sus valiosas sugerencias para mejorar este artículo.

\section{LITERATURA CITADA}

Barker, R.W. 1960. Taxonomic Notes on the Species figured by H. B. Brady in his Report on the Foraminifera dredged by H.M. S. Challenger during the years 1873-1876. Society Economical Paleontological \& Mineral Special Pub. $\mathrm{N}^{\circ}$ 9, 238 pp.

Brady, H. B. 1884. Report on the Foraminifera dredged by H.M.S. "Challenger" during the years 1873 - 1876. Report Voyage Challenger, Zoology, 9:814 pp.

Boltovskoy, E. 1965. Foraminíferos recientes. Eudeba. $510 \mathrm{pp}$

Boltovskoy, E, G. Giussani, S. Watanabe \& R. Wright 1980. Atlas of benthic shelf foraminifera of the southwest atlantic Junk. by. Pub. The Hague- Boston- London. 147 pp.

Clarke K. R. 1993. Non parametric multivatiate analyses of changes in communitiy structure. Australian. Journal Ecology, 18: 117 - 143

Clarke, K. \& R. Gorley 2006. Primer v.6 User Manual/Tutorial PRIMER-E Ltd.: Plymouth Marine Laboratory, 190 pp.

Clarke, K. R. \& R.M. Warwick 2001. Change in marine communities: an aproach to statistical analysis and interpretation. Plymouth Marine Laboratory $44 \mathrm{pp}$.

Dean, W. E. 1974. Determination of carbonate and organic matter in calcareous sediments and sedimentary rocks by loss on ignition comparison with other methods. Journal of Sedimentary Research,Petrology, 44: $242-248$

Decrouez, D. 1989. Generic ranges of Foraminiferida. Revue Paleobiologie, 8 (1): 263 - 321

Ellis, B. \& A. Messina 1944. (et seq.) Catalogue of Foraminifera. American Museum of Natural History Special Pub. 30 vol.

Folk, R. L. 1974. Petrology of sedimentary rocks. Hemphill Publishing Company, Austin, 182 pp. 
Graham D. \& N. Gidgley 2000. Graphical representation of particle shape using triangular diagrams: an excel spreadsheet method. Earth Surface Processes and Landforms, 25: 1473 - 1477

Hromic, T. 1996. Foraminíferos bentónicos (Protozoa: Foraminiferida) de aguas profundas del estrecho de Magallanes, Chile. Anales Instituto Patagonia Serie Cs. Nat. (Chile), 24: 65-86

Hromic, T. 1998. Foraminiferida: Miliolina del estrecho de Magallanes, Chile; Distribución en el área subantártica y antártica. Anales Instituto Patagonia Serie Cs. Nat. (Chile), 26: 107-118

Hromic, T. 1999. Foraminíferos bentónicos de canales australes de canal Kirke, golfo Almte. Montt y seno Última Esperanza, XII ${ }^{a}$ Región, Magallanes y Antártica chilena. Anales Instituto Patagonia Serie Cs. Nat. (Chile), 27: 91-104

Hromic, T. 2001. Foraminíferos bentónicos del canal Baker (47오; 74ํW) Pacífico sudoriental, Chile. Anales Instituto Patagonia Serie Cs. Nat. (Chile), 29: 135-156

Hromic, T. 2002. Foraminíferos bentónicos de bahía Nassau, Cabo de Hornos, Chile. Comparación con foraminíferos del cono sur de América, Antártica y Malvinas. Anales Instituto Patagonia Serie Cs. Nat. (Chile), 30: 95-108

Hromic, T. 2005. Foraminíferos bentónicos entre seno Reloncaví y Golfo Corcovado. Crucero Cimar 10 Fiordos, Informes preliminares, 125-134

Hromic, T. 2007. Biodiversidad y ecología del microbentos (Foraminifera: Protozoa) entre la boca del Guafo y golfo de Penas (43 - 46으) ), Chile. Ciencia y Tecnología del Mar, 30 (1): 89-103

Hromic, T. 2009 a. Estructura comunitaria del taxa Foraminiferida (Protozoa: Foraminiferida) según profundidad, en el fiordo Comau, Chiloé, Chile. Anales Instituto Patagonia (Chile), 37 (1): 39-51

Hromic, T. 2009 b. Distribución batimétrica de foraminíferos bentónicos (Protozoa: Foraminiferida) al sur del estrecho de Magallanes (52음 $-56^{\circ}$ S), Chile. Anales Instituto Patagonia (Chile), 37 (1): 23-38

Hromic, T. 2011 a. Foraminíferos bentónicos recolectados durante la expedición Cimar 14 fiordos, Patagonia chilena. Anales Instituto Patagonia (Chile), 39 (1): 75-89
Hromic, T. 2011 b Foraminíferos bentónicos recientes del estrecho de Magallanes y canales australes chilenos. Cimar 3 Fiordos. Anales Instituto Patagonia (Chile), 39 (2) (en prensa, este volumen)

Hromic, T. 2011 c. Foraminíferos bentónicos de la expedición Cimar 11 Fiordos, Canales Patagónicos chilenos: Biodiversidad y abundancia. Ciencia y Tecnología del Mar, Valparaíso (Chile), 34 (2) (en prensa)

Hromic, T. 2011 d Análisis de la comunidad foraminiferológica bentónica del seno Reloncaví, islas Desertores, Golfo Ancud y Golfo Corcovado, Chile. Ciencia y Tecnología del Mar, 34 (1) (en prensa)

Hromic, T. \& M. Zúñiga 2003. Diversidad, abundancia y distribución de la superfamilia Buliminacea Jones, 1875, en canales y fiordos patagónicos, Pacífico Sudoriental. Anales Instituto Patagonia, Serie Cs. Nat. (Chile), 31: 55-74

Hromic, T. \& M. Zúñiga 2005. Foraminíferos bentónicos de ambientes someros extraídos durante la Expedición Cimar 7 Fiordos, canales patagónicos (42- $47^{\circ}$ S). Boletín de la Sociedad de Biología de Concepción, 75: 25-38

Hromic, T., S. Ishman \& N. Silva 2006. Benthic foraminiferal distribution in Chilean fjords: $47^{\circ} \mathrm{S}$ to $54^{\circ} \mathrm{S}$. Marine Micropaleontology, 59: $115-134$

Ishman, S. \& R. Martínez 1995. Distribution of modern benthic foraminifers from the fjord region of southern Chile ( $42^{\circ} \mathrm{S}$ to $\left.55^{\circ} \mathrm{S}\right)$. Antarctic Journal of the United States, 25: 6-8

Korsum, S. \& M. Hald 2000. Seasonal dynamics of benthic foraminifera in a glacially fed fjord of Svalbard, European Arctic. Journal of Foraminiferal Research, 30 (4): 251-271

Lena, H. 1966. Foraminíferos recientes de Ushuaia (Tierra del Fuego, Argentina). Ameghiniana, 4 (9): 311-336

Loeblich, A. \& H. Tappan 1988. Foraminiferal Genera and Their Classifications. Van Nostrand Reinhold Co. Text- Vol.: 970 pp. Pl. - Vol.: $212 p p+847$

Mackensen, A., G. Schmiedl, J. Harloff \& M. Giese 1995. Deep-sea foraminifera in the South Atlantic Ocean: ecology and assemblage generation. Micropaleontology 41, 342- 
358. fide: Haslett (Eds.) 2002, Quaternary Environmental Micropalaeontology. Oxford University Press 340 pp.

Marchant, M. 1993 Foraminíferos de la Bahía Scholl, Región Magallánica, Chile (Protozoa: Foraminifera). Gayana Zoología, 57 (1): $61-75$

Murray, J. 1973. Distribution and ecology of living benthic foraminiferas. Heineman (Ed.) London $274 \mathrm{pp}$.

Murray, J. 1991. Ecology and Palaeoecology of benthic foraminifera. Longman Scientific \& Technical. 397 pp.

Murray, J. 2006 Ecology and applications of Benthic foraminifera Cambridge University Press $426 \mathrm{pp}$.

Silva N., V. Vidts \& J. Sepúlveda 2001. Materia orgánica, $\mathrm{C}$ y N, su distribución y estequiometría, en sedimentos superficiales de la región central de los fiordos y canales australes de Chile (Crucero CIMAR 2 Fiordos). Ciencia y Tecnología del Mar, 24 (1): 23-40

Sievers, H. \& N. Silva 2008. Waters masses and circulation in austral Chilean channels and fjords. In: N. Silva \& S. Palma (Eds.) 2008. Progress in the Oceanographic Knowledge of Chilean Inner Waters, from Puerto Montt to Cape Horn, Comité Oceanográfico Nacional - Pontificia Universidad Católica de Valparaíso, 53-58

Silva N. 2008. Physical and chemical characteristic of the surface sediments in the austral Chilean channels and fjords. In: N. Silva \& S. Palma (Eds.), Progress in the Oceanographic Knowledge of Chilean Inner Waters, from Puerto Montt to Cape Horn, Comité Ocea- nográfico Nacional - Pontificia Universidad Católica de Valparaíso, 69-75

Silva N. \& P. Ortiz 2002. C y N, su distribución y estequiometría, en sedimentos superficiales de la región sur de la zona de fiordos y canales australes de Chile, 52ㅇ - 56응 S (Crucero Cimar-Fiordo 3). Ciencia y tecnología del Mar, 25 (1): 89-108

Smart, C. 2002. Environmental applications of deep - sea benthic foraminifera. Quaternary Environmental Micropaleontology. In: Haslett (Eds.) 2002, Oxford University Press: 14-58

Schmiedl, C., A. Mackensen \& P.J. Müller 1997. Recent benthic foraminifera from the eastern South Atlantic Ocean: dependance on food supply and water masses. Marine Micropaleontology, 32: 249 - 287 fide Haslett (Eds.) 2002, Quaternary Environmental Micropalaeontology. Oxford University Press (England). 340 pp.

Violanti, D., B. Loi \& R. Melis 2000. Distribution of Recent Foraminifera from the Strait of Magellan. First quantitative data. Bolletino Museo Regional Scientia Naturalli, 17 (2): 511-539

Zapata, J. A. \& R. Alarcón 1988. Foraminíferos bentónicos del Estrecho de Magallanes (52 $2^{\circ} 33^{\prime}$ S; 6954' W), Chile. Biota, 4: 17-29

Zapata, J. \& H. Moyano 1997 Foraminíferos bentónicos recientes de Chile Austral. Boletín de la Sociedad Biología, 68: 27-37

Zapata, J., C. Zapata \& A. Gutierrez 1995. Foraminíferos bentónicos del sur de Chile. Gayana Zoología, 59 (1): 23-40

Zar, J. H. 1996. Biostatistical analysis. Prentice Hall, $888 \mathrm{pp}$. 
Anexo 1: Lista sistemática de los foraminíferos bentónicos de seno Gallegos y bahía Brookes y su abundancia relativa

\begin{tabular}{|c|c|c|c|c|}
\hline Suborden & Familia & Género & Especie & $\mathrm{N} \%$ \\
\hline \multirow[t]{18}{*}{ TEXTULARIINA } & Rhabdamminidae & Rhabdammina & Rhabdammina abyssorum Sars, 1869 & 5 \\
\hline & \multirow[t]{2}{*}{ Ammodiscidae } & \multirow[t]{2}{*}{ Ammodiscus } & Ammodiscus tenuis Brady, 1884 & 0,1 \\
\hline & & & Ammodiscus flavidus Höglund, 1947 & 1,3 \\
\hline & \multirow[t]{4}{*}{ Hormosinidae } & \multirow[t]{4}{*}{ Reophax } & Reophax filiformis Sars, 1872 & 0,6 \\
\hline & & & Reophax pilulifer Brady, 1884 & 2,4 \\
\hline & & & Reophax scorpiurus Montfort, 1808 & 1 \\
\hline & & & Reophax sp. 1 & 0,2 \\
\hline & \multirow[t]{4}{*}{ Haplophragmoididae } & \multirow[t]{4}{*}{ Labrospira } & $\begin{array}{l}\text { Labrospira jeffreysii (Williamson) =Nonionina } \\
\text { jeffreysii Williamson, } 1858\end{array}$ & 12,6 \\
\hline & & & $\begin{array}{l}\text { Labrospira crassimargo (Norman) } \\
=\text { Haplophragmoides crassimargo Norman, } 1892\end{array}$ & 0,1 \\
\hline & & & Labrospira kosterensis Höglund, 1947 & 2,1 \\
\hline & & & Labrospira sp. 1 & 4,3 \\
\hline & \multirow[t]{4}{*}{ Lituolidae } & \multirow{4}{*}{ Ammobaculites } & Ammobaculites americanus Cushman, 1910 & 10,5 \\
\hline & & & Ammobaculites sp. 1 & 0,2 \\
\hline & & & Ammobaculites sp. 2 & 0,2 \\
\hline & & & Ammobaculites sp. 3 & 0,1 \\
\hline & Spiroplectamminidae & Morulaeplecta & Morulaeplecta bulbosa Höglund, 1947 & 3,9 \\
\hline & Remaneicidae & Remaneica & Remaneica helgolandica Rhumbler, 1938 & 0,6 \\
\hline & Trochamminide & Trochammina & Trochammina discorbis Earland, 1934 & 0,9 \\
\hline \multirow[t]{10}{*}{ ROTALIINA } & Cassidulinidae & Cassidulinoides & $\begin{array}{l}\text { Cassidulinoides parkerianus (Brady) = } \\
\text { Cassidulina parkeriana Brady, 1884, }\end{array}$ & 0,2 \\
\hline & Buliminidae & Globobulimina & $\begin{array}{l}\text { Globobulimina notovata (Chapman) }= \\
\text { Bulimina notovata Chapman, } 1941\end{array}$ & 16,8 \\
\hline & \multirow[t]{3}{*}{ Uvigerinidae } & \multirow[t]{2}{*}{ Uvigerina } & Uvigerina bifurcata d'Orbigny, 1839 & 3,5 \\
\hline & & & Uvigerina sp. 1 & 0,1 \\
\hline & & Angulogerina & $\begin{array}{l}\text { Angulogerina angulosa (Williamson)= } \\
\text { Uvigerina angulosa Williamson, } 1858 .\end{array}$ & 0,9 \\
\hline & Cibicididae & Cibicides & Cibicides sp. 1 & 0,1 \\
\hline & Trichohyalidae & Buccella & $\begin{array}{l}\text { Buccella peruviana (d'Orbigny) = Rotalina } \\
\text { peruviana d'Orbigny, } 1839\end{array}$ & 2,8 \\
\hline & \multirow[t]{3}{*}{ Nonionidae } & Nonion & $\begin{array}{l}\text { Nonion scaphum (Fichtel \& Moll) }= \\
\text { Nonionina scapha Fichtel \& Moll, } 1803\end{array}$ & 8,6 \\
\hline & & Nonionella & $\begin{array}{l}\text { Nonionella auris (d'Orbigny) = Valvulina } \\
\text { auris d'Orbigny, } 1839\end{array}$ & 18,8 \\
\hline & & Pullenia & $\begin{array}{l}\text { Pullenia subcarinata (d'Orbigny) = Nonionina subcarinata } \\
\text { d'Orbigny, } 1839 \text { = Pullenia quinqueloba (Reuss), } 1851\end{array}$ & 0,3 \\
\hline \multirow[t]{6}{*}{ LAGENINA } & \multirow[t]{4}{*}{ Lagenidae } & \multirow[t]{4}{*}{ Lagena } & $\begin{array}{l}\text { Lagena caudata (d'Orbigny) =Oolina } \\
\text { caudata d'Orbigny, } 1839 .\end{array}$ & 0,6 \\
\hline & & & Lagena hispidula Cushman 1913 & 0,3 \\
\hline & & & $\begin{array}{l}\text { Lagena striata (d'Orbigny) = Oolina } \\
\text { striata d'Orbigny, } 1839\end{array}$ & 0,4 \\
\hline & & & Lagena sulcata Walker \& Jacob, 1798 & 0,1 \\
\hline & Ellipsolagenidae & Fissurina & Fissurina sp. 1 & 0 \\
\hline & Glandulinidae & Glandulina & Glandulina communis d'Orbigny 1826 & 0,3 \\
\hline MILIOLINA & Hauerinidae & Quinqueloculina & $\begin{array}{l}\text { Quinqueloculina seminula (Linnaeus) = } \\
\text { Serpula seminula Linnaeus, } 1758\end{array}$ & 0,9 \\
\hline
\end{tabular}

